\section{Covid restrictions tighten as omicron cases double every two to three days}

\author{
Ingrid Torjesen
}

"Plan B" restrictions will be implemented in England from 10 December in an attempt to slow the spread of the omicron variant, which is now present in every part of the UK, with case numbers doubling every two to three days.

The prime minister announced the measures, which include mandatory mask wearing in most public indoor settings, vaccine passports for large capacity events, and working from home, during a televised press conference on 8 December. Although the measures were "irritating," Boris Johnson said, they were the best way to ensure a "Christmas as close to normal as possible," given that omicron seemed to be more transmissible than the previous delta variant and case numbers were "growing much faster."

And with covid hospital admissions in South Africa doubling in a week, "we can't yet assume omicron is less severe than previous variants," Johnson added.

Although working from home is being encouraged, people will still be able to socialise freely, including at work Christmas parties.

Chaand Nagpaul, the BMA's chair of council, said that the government's decision to implement Plan B was "the right one."

"We must not risk complacency by ignoring the rapid doubling of omicron cases every two to three days, which may result in hospital admissions in the weeks to come," he said. "This threatens to overwhelm the health service and could have a major impact on the NHS's ability to cope this winter.”

A risk assessment by the UK Health Security Agency said that omicron's "significant growth advantage" over delta meant that it was likely to outcompete delta in the UK and become the dominant variant within the next two to four weeks if it continued to spread at its current pace. The agency also confirmed that previous infection or vaccination offered reduced protection against omicron, in comparison with delta, but that data were as yet insufficient to quantify the effect of this on the risk of reinfection and severe disease.

As at 8 December a total of 568 confirmed cases of the omicron variant had been reported in the UK, a rise of 131 on the previous day. The UKHSA figures show that 448 of these cases were in England, 108 in Scotland, nine in Wales, and three in Northern Ireland.

The omicron variant carries a specific genetic characteristic called the $S$ gene dropout, which suggests but does not confirm the variant and is shown in the PCR test result. Although the characteristic is seen with some other variants, such as alpha, it is not seen with delta so can be used as a proxy for tracking the spread of omicron and highlighting potential cases that can be confirmed by genetic sequencing. Around half the PCR tests used in the UK are able to detect $\mathrm{S}$ gene dropout.

The number of PCR tests showing $S$ gene dropout rose to 705 in the week beginning 30 November, when it had routinely been fewer than 150 cases a week (less than $0.1 \%$ of cases) in previous weeks. Most cases showing $\mathrm{S}$ gene dropout are in London and the South East of England.

Scientists have said that there may also be a "stealth" version of omicron that does not carry the S gene dropout, ${ }^{1}$ which would make it more difficult to track the spread of the variant and increase in case numbers. Seven cases of the stealth variant have been identified in South Africa, Australia, and Canada, but it may have spread more widely.

The UK's vaccine booster programme was extended to over 40 from 8 December, and the time from second dose to booster cut from six to three months.

\section{Reduced immunity}

Results from a small South African study, which has yet to be peer reviewed and is available as a preprint, indicate that omicron may circumvent some of the antibody immunity produced by the Pfizer vaccine, ${ }^{2}$ and this has been backed by data from Pfizer and BioNTech. ${ }^{3}$

The South African study found a 41-fold drop in antibodies' virus blocking ability in some of the samples from 12 double vaccinated people, when compared with those generated against the beta strain, which was also first detected in South Africa, in 2020.

Paul Moss, professor of haematology and deputy head of the University of Birmingham's College of Medical and Dental Sciences, said this suggested that only $2.5 \%$ of the antibody neutralisation activity was retained.

However, virus neutralisation was much higher in five samples from people who had previously been infected with SARS-CoV-2. Jonathan Ball, professor of molecular virology at the University of Nottingham, said, "This group effectively mimics what we would expect in people who had had two doses of vaccine plus a boost."

He added, "While this reduced antibody immunity might mean more people can be infected and have mild symptoms, I am still confident that the vaccines, especially after a boost, will still protect from serious disease.”

Initial data from Pfizer and BioNTech showed that although a double dose and a booster produced levels 
of neutralisation against omicron similar to those of two doses of vaccine against the original SARS-CoV-2 virus, there was a more than 25 -fold reduction in neutralisation in people who had only been double vaccinated. ${ }^{3}$

1 Sample I, Walker P. Scientists find "stealth" version of Omicron that may be harder to track. Guardian.7 Dec 2021. https://www.theguardian.com/world/2021/dec/07/scientists-find-stealthversion-of-omicron-not-identifiable-with-pcr-test-covid-variant.

2 Sele S, Jackson L, Khan K, etal. SARS-CoV-2 omicron has extensive but incomplete escape of Pfizer BNT162b2 elicited neutralization and requires ACE2 for infection.https://www.ahri.org/wpcontent/uploads/2021/12/MEDRXIV-2021-267417v1-Sigal.pdf.

3 Pfizer. Pfizer and BioNTech provide update on omicron variant. Dec 2021. https://www.pfizer.com/news/press-release/press-release-detail/pfizer-and-biontech-provide-update-omicronvariant.

This article is made freely available for use in accordance with BMJ's website terms and conditions for the duration of the covid-19 pandemic or until otherwise determined by BMJ. You may use, download and print the article for any lawful, non-commercial purpose (including text and data mining) provided that all copyright notices and trade marks are retained. 